\title{
Artificial Neural Networks and their Applications in Modern Networks Technology
}

\author{
Abdul Basit ${ }^{1 *}$, Iftikhar Alam Khan ${ }^{2}$, Zainab Mohammad Aslam ${ }^{3}$, Arshiya Subhani ${ }^{4}$ \\ 1, 2, 3, 4 Department of Creative Computing, Bath Spa University Academic Center RAK, Ras Al-Khaimah, UAE
}

\section{Keywords \\ Neural networks \\ Modern networks \\ Wireless network \\ Information processing}

Received: 21 March 2021

Accepted: 29 May 2021

Published: 7 June 2021

\begin{abstract}
The purpose of this chapter is to introduce a powerful class of mathematical models: the artificial neural networks. This is a very general term that includes many different systems and various types of approaches, both from statistics and computer science. The analogy is not very detailed, but it serves to introduce the concept of parallel and distributed computing. Neural networks explain and give the proper demonstration for the applications of neural networks and wireless network. The artificial network and wireless network have strong connection and can be investigated and explained properly. There are also some models which are called mathematical models which can explain and demonstrate the artificial and wireless networks in detail. There are many organizations which are using artificial neural networks by using sensor technology for wide range of purpose. Then we analyze in detail a widely applied type of artificial neural network: the feed-forward network with error back-propagation algorithm. We illustrate the architecture of the models, the main learning methods and data representation, showing how to build a typical artificial neural network. Our aim is not to examine them all (it would be a very long discussion), but to understand the basic functionality and the possible implementations of this powerful tool. We initially introduce neural networks, by analogy with the human brain.
\end{abstract}

(c) 2021 The Author(s). Published by TAF Publishing.

\section{INTRODUCTION}

Artificial neural network can be applied to various fields and has very broad range of tasks in many different disciplines. The main focus is on those areas which are very close to the potential networking system of neural networks and wireless network system. There are some applications which give the arbitrary solution. There are some same characteristics which can categories neural networks classification in different problems. The main ability of neural network structure is to perform different universal functions like ability to form and decide complex decisions on the bases of generalizations [1]. There are some capabilities of importance in virtually models of neural networks which can be derived from observed behavior and constraints. Artificial neural network system is information processing system which designed and inspired like as the nervous system [2]. This works as like human brain processes the information for different types of inputs in biological way. The main key element is for this system, which is called information system of different types networking system [3]. This system exists between the biological and synaptic connections of neurons. In wireless technology, computer network and data network based on different systems like different types of wires, switches, routers and wireless systems. In computer network system, all the computer systems and network devices pass through data from different states to other devices for connectivity and sharing of data [4]. The connections are established with wires and wireless technology system with devices of networks. These connections are established with nodes, clients or server computers and dedicated servers are also used for sharing, sending and receiving of data. This system comprises over on many highly interconnected elements like neurons. These elements solved different problems. The ANN and wireless technology system configure different specific application and problems according to the recognition of pattern sys-

\footnotetext{
${ }^{*}$ Corresponding author: Abdul Basit

†email: abdul@bathspa.ae
} 
tem and different types of classification of data. There are many similarities of neural network and human body structure network which detects different types of errors and sort out the problem and errors very carefully. The neural network applications have existing problems in different fields $[5,6]$.

\section{A. ANN Theory and Models and Research Objectives}

Different type of Neural Networks has been proposed but all of them have three things in common: the individual neuron, the connection between them (architecture), and the learning algorithm. Each type restricts the kind of connections that are possible. For example, it may specify that if one neuron is connected to another, then the second neuron cannot have another connection towards the first.

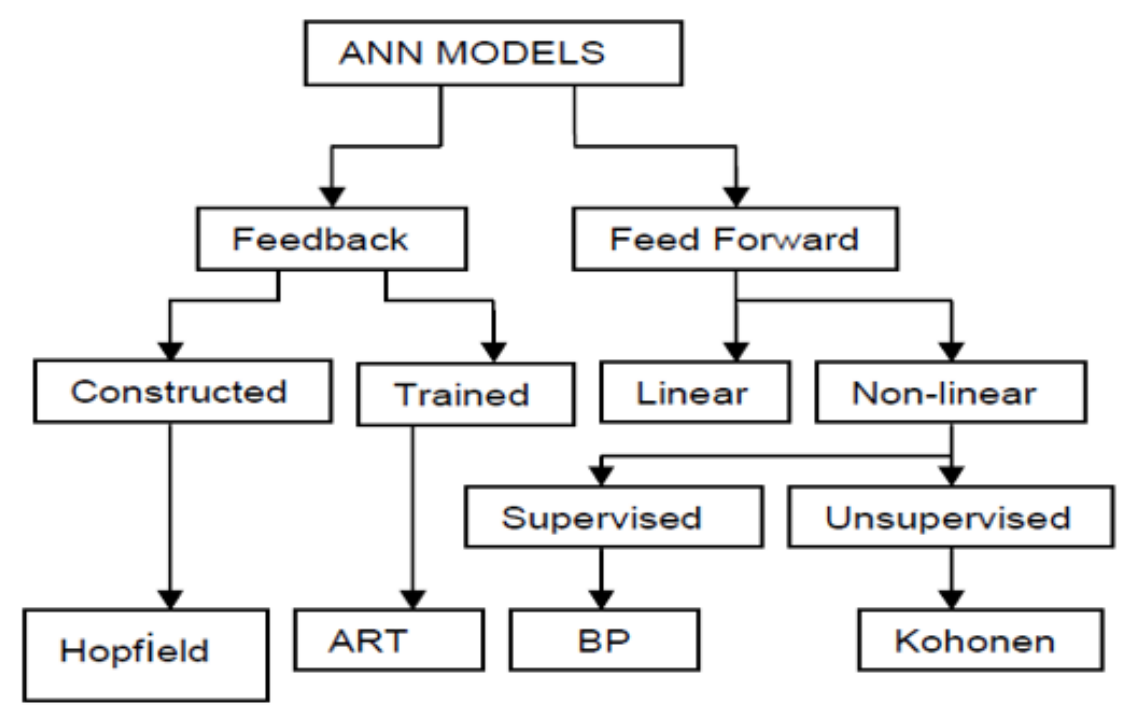

Fig. 1. General classification of ANN models

Shows a general classification of ANN models. These devices are made to talk with each other for communication purpose. Early system was used to send telegraphs and telephone has invented from these types of things for communication. Early devices for communications were different and complicated for passing the data but now days the system is completely changed [7]. The developers have connected the computers system in such a way that these computers can use different resources, sharing of data and transferring of data worldwide in different formats. The new system was introduced as communication protocol which is known as data packet switching with number of applications like voice transmissions and other protocols [8].

\section{B. Objectives}

1. Parallel processing can perform more than one tasks at the same time over the network.

2. All information stored on ANN network not only on database.

3. This model helps in understanding the non-linear complex relationship with human brain.

Requirement engineering models mainly presents professional projects of managers, software architecture of sys- tem developers and software engineers can take benefit from these models to produce new things and structures of latest innovation for future. Requirement engineering models sometimes depend on attributes which help in developing the software. In requirement engineering models the most critical and complex process for the development of technical systems $[9,10]$. So this model helps in describing the multidisciplinary roles of software requirement engineering are vital in recognizing the patterns for social interaction between different models of engineering. The main reason in software developing model is requirement engineering process has most important and dominant effect of capabilities of resulting products by software engineering [11]. Furthermore software engineering is a process of diversifying the products which demands from the most diversified set of stakeholders. There is another reason which makes the project complicated and difficult I requirement engineering models. Requirement engineering models is a systematic approach towards through software engineering collects requirement from several sources and these sources are implemented in development process $[12,13]$. 


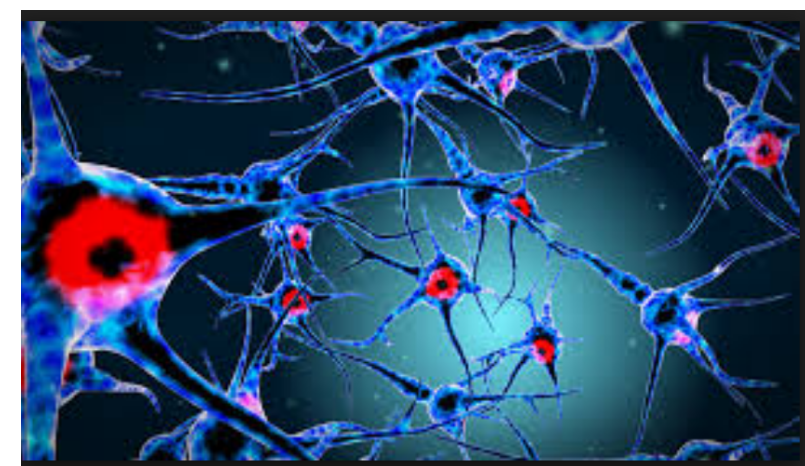

Fig. 2. neurons and neural structure

Requirement engineering basically activities cover the whole system and software development cycle with the implementation of different sources. Mainly the software engineering is iterative process of models. However in large and complex companies structure which get the support of developing the accurate requirements which are helpful in developing the software strategies. In this way, these strategies remain consistent until several years and month [14]. Requirement engineering models development has been realized to be impossible practices and iterative process of software engineering structures with other activities of design and coding phase.

\section{METHODOLOGY}

There are some rules which are called firing rules and these rules have important concept in neural network and wireless system. These rules give the calculations method for getting input and output of the system [15]. This system maintains and relates to the neural network system. WLAN these rules can create the hamming distance in the network. There is collection of training patterns for different nodes and cause to fire the input patterns these patterns are not in the collection list. They have lot of input elements in the list and they are very close to the pattern taught to the indefinite state. There are three inputs which are taught to the output for different links of the $\mathrm{x} 1$ and $\mathrm{x} 2$. There are more inputs called 111 and 101 to the 0 input and the inputs 000 or more than this range [16].

TABLE 1

WIRELESS NETWORK SYSTEM CALCULATIONS METHOD

\begin{tabular}{lllllllll}
\hline \hline $\mathrm{X} 1$ & 0 & 0 & 1 & 1 & 0 & 0 & 0 & 0 \\
$\mathrm{X} 2$ & 0 & 0 & 0 & 1 & 1 & 0 & 0 & 0 \\
$\mathrm{X} 3$ & 1 & 1 & 1 & 0 & 0 & 1 & 1 & 0 \\
In out & 0 & 1 & 1 & 1 & 0 & 1 & 0 & 0 \\
\hline \hline
\end{tabular}

TABLE 2

WIRELESS NETWORK SYSTEM CALCULATIONS METHOD INPUT AND OUTPUT

\begin{tabular}{lllllllll}
\hline X1 & 1 & 1 & 0 & 0 & 1 & 1 & 0 & 0 \\
X2 & 1 & 1 & 0 & 0 & 0 & 1 & 1 & 1 \\
X3 & 0 & 0 & 0 & 1 & 1 & 0 & 1 & 0 \\
In out & 0 & 1 & 1 & $0 / 1$ & $1 / 0$ & 1 & 0 & 0 \\
\hline \hline
\end{tabular}

Artificial neural network is connected to the device for getting inputs and outputs form the network structure. There are two modes of operation can be found in neuron structure [17]. These modes are classified as training mode and using mode. Neurons can fire particulars pattern for the network in training mode but in using mode neurons can fired detected inputs. There are all events of neural network belong to the training and using session.

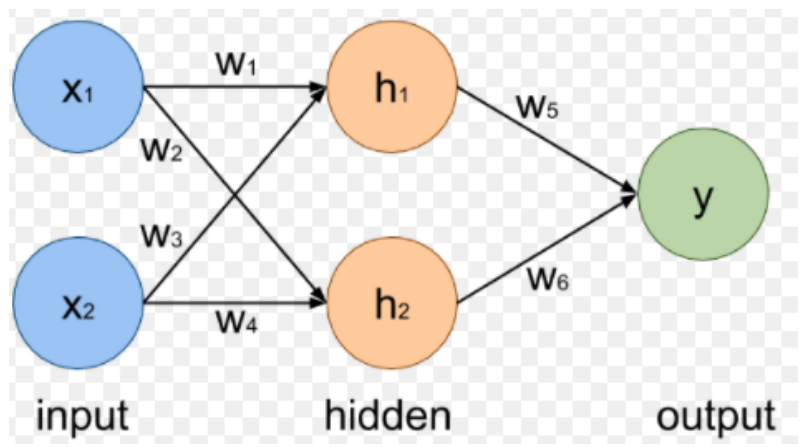

Fig. 3. Neural network's mathematical notation

\section{A. Pattern Recognition in Neural Network System}

There is an important feature of neural network system is called pattern recognition. This pattern can be used by using feed forward. Neural network is designed according to the training system [18]. The network is designed to get the inputs from the system. Network is designed to receive the outputs from pattern. When neural network is used it gets the inputs from the pattern and then shows the response behavior according to that input behavior. The power of artificial network comes to true when it uses inputs [19]. The neural network gets the input and use the taught pattern to recognize the pattern and sensors.

\section{FINDINGS AND DISCUSSION}

There are capabilities of ultra wide bands. These are endless in network system. This ultra wide band can achieve the data rates separately. This can be in the range of 100 to 50 Mbps. So these data rates can easily be achieved. The transmission rate of the data rates in network can be different from different range of ratio between 2 to 10 meters long. This is because of the strict limitations of the system network in neural network system [20]. The ultra violet can achieve the limitations of the network. The capacity of the 
network can also be achieved. When the transmission range is less than 10 to 12 meters, this seriously affects the network system and the whole network system becomes detectable in sensor. If the transmission is higher than 12 then the frequency of the network is changed and protocols start moving from low to higher level [21].

\section{A. Recognition of Network System in Artificial Network}

This network is designed to accept the patterns which are called $\mathrm{T}$ and $\mathrm{H}$. The all associated patterns are called black and other called simulators [22].

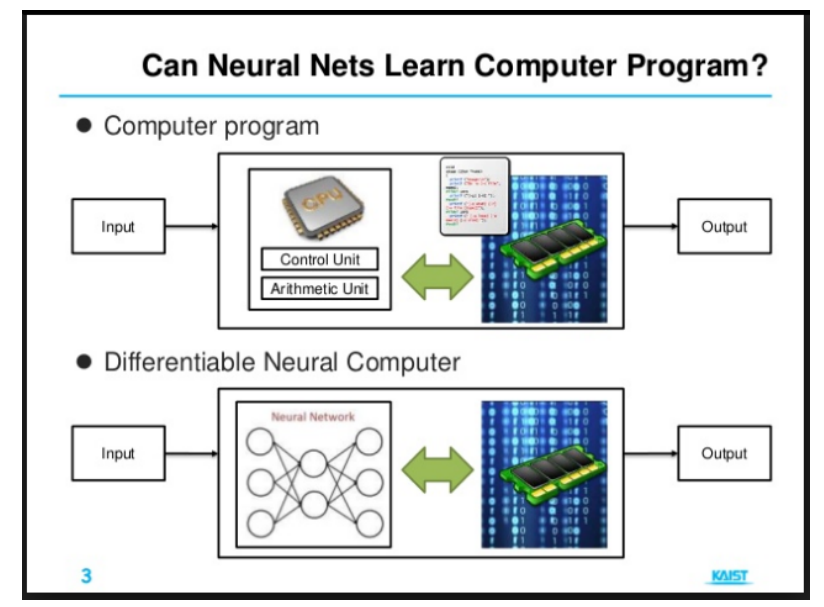

Fig. 4. Neural nets

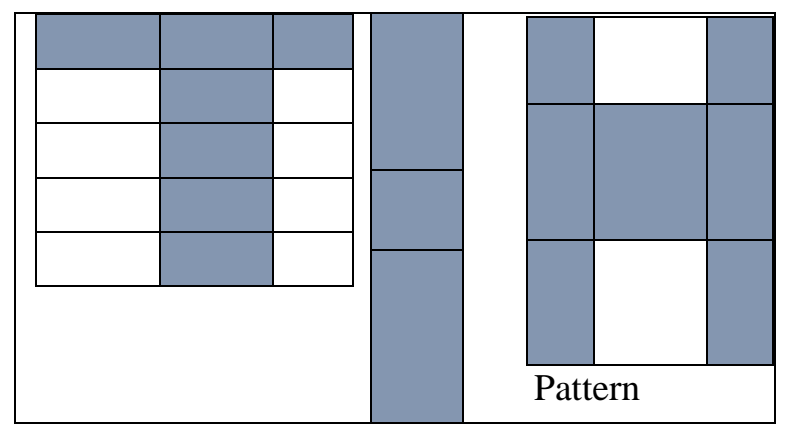

Fig. 5. Pattern input output

\section{B. Comparison and Decision in Neural Network and Wireless Sensors}

There are some features which are relevant to the features of blue tooth and RF system. These are detected by protocols. These protocols are separately assigned to the network system. The data from this table move from one location to the other location [23]. There are many factors which can affect the data transmission. The data can make the final decision from one place to the other in graph. WPAN All the factors are going in proper direction and give excellent advantage. All protocols give best work to the factors of the final decision. The all factors of protocols are weighed in discussion [24].

\section{Complicated Structure of Neuron and Artificial Net- work}

The previous neuron system does not support the conventional computers and these computers can not delay in working. A more sophisticated is developed for the neural network system. In previous system, the inputs are weighted and each decision is taken on the base of the sensor [25]. The weight of different inputs is complicated and can be designed to be multiplied. These all weights are added together in the system to receive the proper signals from the network system. These are the other conditions where neurons are not set to transmit [26].

\section{MCP Neuron Structure and System of Neural Network System}

The addition of network system and input weights to make it threshold structure of the neuron network system to classified the structure of the neural network system. The neuron related to the MCP has the ability to share and denotes the particular system in network system. The former system is used to forward the feedback network system in wireless network structure [27].

\section{E. Network Detection Frequency Shift Keying in Neural and Wireless Network System (FSK)}

FSK is digital modulation method and technique which can be similar in frequency and modulation of FM. This supports to analog devices. But this is the main difference between FSK and FM. Each digital symbol has different frequencies of 101 for FSK. This is the map which can specify the frequency properly. The main and big advantage is that this FSK assigns the symbols in network [28].

\section{F. Neural System and Orthogonal Frequency Division Multiplexing in Network and Wireless System of Neu- ral Network (OFDM)}

The main task of OFMD is to divide the modulation parts in bandwidth into smaller bandwidth of modulation. This can carries the frequencies that close to the orthogonally in the network system. This bandwidth of OFDM consist single set of transmitted data. This can transmit the data into different smaller parts [29].

\section{G. Neural Network and Wireless Techniques for Phase Shift Keying (PSK)}

This is another technique that is similar to the modulation phase that communicates with the system in modulation. 
There are finite set of phases that maps in specific bit rate [30]. This binary map bit key can map to the keys to the zero value. There are two main symbols of BPSK.

\section{H. Artificial Neural networks and Wireless Network Comparison of Conventional Computers}

Neural network can deal with different and different approaches to sort out the problems and networking system. Conventional computers are used I networking and artificial intelligence. Computers receive the instructions to handle different types of instructions so that machine will execute the instructions properly and process data [31]. There is problem in doing the specific steps and execute the steps in different ways. Sometimes computer can follow the steps and execute the instructions properly. There are some conventional computers which can support the systems in networks. The computers can sort different conflict issues between the systems and the other servers which are connected with that [32]. There are some restricted problems which can sort out the problems quickly. There are some computers which can be more useful and can better handle the network security and structure of the networking system. The problems with networking and the servers are the main problem of lot of users at the same time [28]. The neural network can process all the information as our human brain is processing the information.

\section{Neural network Proposed Solution and Wireless Fac- tors}

DCS system is used to monitor the network system by wires. It helps in finding the locations of the networks. Bu this system cannot be used in wireless technology. This system is required to connect the devices with wires in network system. This system is inefficient to trace out the faults which are presents in the network and would not be able to find collision detection in the network. (WEP) If the network is accessed by the users this gives inefficiency and the network system is stuck [15]. To sort out the problem this system is fully monitored by wireless system. The all devices are capable of connecting with the wireless network. This thesis is concerned with the development of neural network system to find different errors and problems form the network along with WLAN and WAN system [26]. When this system is connected the wired system is switched to the wireless system and the wireless system can be used to monitor for the entire network. Once the wireless network system is integrated and the neural network system helps in finding the errors by collision detection. There is no interference of different types of collision and the network works smoothly [22]. Protocol system is also introduced in neural network system to make the network system reliable and efficient wireless connection. It runs smoothly the network system of the sensors. There are many multiple devices in the specific area and can be efficient in the network. A decision is made efficient to detect the sensor system 40 .

\section{J. Feedback Network System in Artificial Network Sys- tem}

Feedback system has different networking system and platform. These signals travel in both directions. Feedback system in networks is powerful and can sort out extreme complication. These feedback systems are dynamic their state and direction changing continuously until it reaches at certain point. Feedback system is architecture of interactive and recurrent system of the denote feedback [17].

\section{K. Supervised Learning in Neural Network}

There is an external teacher who will control the output unit system in network system. There is learning process in the unit which can give the response according to the network sensors. Global information is required I the system. There are errors in the system which can move the errors. There is an important concerning issue which can lead to the desired computed system.

\section{Unsupervised Learning in Neural Network System}

There is no external teacher who will do on the bases of local information. This is called self organization information of the system. Data is represented to the network system for setting network [20].

\section{Transferring Function in Neural Network}

The system of neural network is based on artificial network system. This is only specified for the network. This project falls in three categories in neural network. The names of three categories are linear, threshold and sigmoid. There are some linear units. The linear units give different activities and output. (PDA) This depends on the total weighted output system. In three units system, the output is set for the three levels. These three levels are different and same. The sigmoid units can give output.

\section{N. Example to Show the Training Procedure in Net- working System}

The network is assumed to recognize the digits of the networking. In this system an array is used to detect the sensors in network system. The network needs more sensors to detect the networking system. This type of network needs 
256 inputs and then these inputs will be given outputs by detecting sensors. These sensors record different range of values from on section to the other section and this section will support the network properly. This kind of digit records the reading of the sensors

\section{o. The Background Propagation of Algorithm in Neural Networks and Wireless}

There are some tasks in which neural networks perform some training costs. The each unit is given the errors between description and output. The actual output is reduced. This network is required to reduce the network problem from the network system. This system is also known as the error derivative system of the EW process.

\section{P. Main Applications of Neural Networks and Wireless Network Practice of Neural Networks and Wireless Network System}

There is complete description of the neural network handling system. How the real world applications are suited for the network. The neural network system has its broad application to control the entire network system. This application can solve the real world problems and applications. Neural network system is applied to the many organizations and industries. So this can be applied to many network industries. Neural networks are best to identifying different patterns and trends in data industry I which they are suited for the prediction of the needs [33].

\section{CONCLUSION AND FUTURE WORK}

There is parallel system run with the support of real time. Neural networks can also contribute to those areas in which research is done like neurology and other departments. They are regularly using different models of models parts in living organisms. The internal mechanism can be easily investigated. The neural network is most inspective and network created system which deals different sensors. The neural network system can automatically detect sensors and pass the data to other nodes of the server. Most exciting part is conscious area of network which can produce mechanical property. So the finally I would like to explain more terms and details of the network system which can produce huge network system in wireless and other network servers. These networks also promote the fuzzy logic and other related networks. In wireless technology, computer network and data network based on different systems like different types of wires, switches, routers and wireless systems. In computer network system, all the computer systems and network devices pass through data from different states to other devices for connectivity and sharing of data [4]. The connections are established with wires and wireless technology system with devices of networks. These connections are established with nodes, clients or server computers and dedicated servers are also used for sharing, sending and receiving of data.

\section{REFERENCES}

[1] M. Haseeb, L. W. Mihardjo, A. R. Gill, K. Jermsittiparsert et al., "Economic impact of artificial intelligence: New look for the macroeconomic assessment in Asia-Pacific region," International Journal of Computational Intelligence Systems, vol. 12, no. 2, pp. 1295-1310, 2019. doi: https://doi.org/10.2991/ijcis.d.191025.001

[2] V.Van Roy et al., "Ai watch-national strategies on artificial intelligence: A European perspective in 2019," Joint Research Centre, Ispra, Italy, JRC working papers JRC119974, 2020.

[3] E. Garfield, "Citation analysis as a tool in journal evaluation," Science, vol. 178, no. 4060, pp. 471-479, 1972.

[4] A. Van Raan, "The use of bibliometric analysis in research performance assessment and monitoring of interdisciplinary scientific developments," TATuP-Zeitschrift für Technikfolgenabschätzung in Theorie und Praxis, vol. 12, no. 1, pp. 20-29, 2003.

[5] Deloitte. (2020) The socio-economic impact of AI in healthcare. [Online]. Available: https://bit.ly/3KM85nG

[6] N. Z. T. Abdulnabi and O. Altun, "Batch size for training convolutional neural networks for sentence classification," Journal of Advances in Technology and Engineering Studies, vol. 2, no. 5, pp. 156-163, 2016. doi: https://doi.org/10. 20474/jater-2.5.3

[7] J. Mokhnacheva and I. Mitroshin, "Nanoscience and nanotechnologies at the Moscow domain: A bibliometric analysis based on Web of Science (Thomson Reuters)," Information Resources of Russia, vol. 6, pp. 17-23, 2014.

[8] K. Debackere and W. Glänzel, "Using a bibliometric approach to support research policy making: The case of the Flemish BOF-key," Scientometrics, vol. 59, no. 2, pp. 253-276, 2004. doi: https://doi.org/10.1023/b:scie.0000018532. 70146.02

[9] H. F. Moed, "The effect of "open access" on citation impact: An analysis of ArXiv's condensed matter section," Journal 
of the American Society for Information Science and Technology, vol. 58, no. 13, pp. 2047-2054, 2007. doi: https://doi. org/10.1002/asi.20663

[10] R. Suryanita, H. Maizir, and H. Jingga, "Prediction of structural response based on ground acceleration using artificial neural networks," International Journal of Technology and Engineering Studies, vol. 3, no. 2, pp. 74-83, 2017. doi: https: //doi.org/10.20469/ijtes.3.40005-2

[11] T. U. Daim, G. R. Rueda, and H. T. Martin, "Technology forecasting using bibliometric analysis and system dynamics," in A Unifying Discipline for Melting the Boundaries Technology Management. Portland, OR: IEEE, 2005. doi: https://doi. org/10.1109/PICMET.2005.1509681

[12] T. Inaba and M. Squicciarini, "ICT: A new taxonomy based on the international patent classification," Organisation for Economic Co-operation and Development, Paris, France, Tech. Rep., 2017.

[13] E. Valarezo, P. Rivera, J. Park, G. Gi, T. Kim, M. Al-Antari, M. Al-Masni, T. Kim et al., "Human activity recognition using a single wrist IMU sensor via deep learning convolutional and recurrent neural nets," Journal of ICT, Design, Engineering and Technological Science1, vol. 1, no. 1, pp. 1-5, 2017. doi: https://doi.org/10.33150/JITDETS-1.1.1

[14] L. Egghe, "Dynamic h-index: The hirsch index in function of time," Journal of the American Society for Information Science and Technology, vol. 58, no. 3, pp. 452-454, 2007. doi: https://doi.org/10.1002/asi.20473

[15] R. Rousseau and F. Y. Ye, "A proposal for a dynamic h-type index," Journal of the American Society for Information Science and Technology, vol. 59, no. 11, pp. 1853-1855, 2008. doi: https://doi.org/10.1002/asi.20890

[16] A. F. Van Raan, "Fatal attraction: Conceptual and methodological problems in the ranking of universities by bibliometric methods," Scientometrics, vol. 62, no. 1, pp. 133-143, 2005. doi: https://doi.org/10.1007/s11192-005-0008-6

[17] R. I. Muhamedyev, R. M. Aliguliyev, Z. M. Shokishalov, and R. R. Mustakayev, "New bibliometric indicators for prospectivity estimation of research fields," Annals of Library and Information Studies, vol. 65, pp. 62-69, 2018.

[18] B. Copeland. (2021) Artificial intelligence. [Online]. Available: https://bit.ly/3AzL5Dq

[19] M. Mills. Artificial intelligence in law: The state of play 2016. [Online]. Available: https://bit.ly/3tUNyY2

[20] G. Nguyen, S. Dlugolinsky, M. Bobák, V. Tran, Á. L. García, I. Heredia, P. Malík, and L. Hluchỳ, "Machine learning and deep learning frameworks and libraries for large-scale data mining: A survey," Artificial Intelligence Review, vol. 52, no. 1, pp. 77-124, 2019. doi: https://doi.org/10.1007/s10462-018-09679-z

[21] J. A. Cruz and D. S. Wishart, "Applications of machine learning in cancer prediction and prognosis," Cancer Informatics, vol. 2, pp. 59-77, 2006. doi: https://doi.org/10.1177/117693510600200030

[22] R. Miotto, F. Wang, S. Wang, X. Jiang, and J. T. Dudley, “Deep learning for healthcare: Review, opportunities and challenges," Briefings in Bioinformatics, vol. 19, no. 6, pp. 1236-1246, 2018. doi: https://doi.org/10.1093/bib/bbx044

[23] P. J. Ballester and J. B. Mitchell, “A machine learning approach to predicting protein-ligand binding affinity with applications to molecular docking," Bioinformatics, vol. 26, no. 9, pp. 1169-1175, 2010.

[24] M. S. Mahdavinejad, M. Rezvan, M. Barekatain, P. Adibi, P. Barnaghi, and A. P. Sheth, "Machine learning for internet of things data analysis: A survey," Digital Communications and Networks, vol. 4, no. 3, pp. 161-175, 2018. doi: https: //doi.org/10.1016/j.dcan.2017.10.002

[25] G. Abramo, C. D’Angelo, and F. Pugini, 'The measurement of Italian universities' research productivity by a non parametric-bibliometric methodology," Scientometrics, vol. 76, no. 2, pp. 225-244, 2008. doi: https://doi.org/10.1007/ s11192-007-1942-2

[26] C. R. Farrar and K. Worden, Structural Health Monitoring: A Machine Learning Perspective. New York, NY: John Wiley \& Sons, 2012.

[27] J. Lai, J. Qiu, Z. Feng, J. Chen, and H. Fan, "Prediction of soil deformation in tunnelling using artificial neural networks," Computational Intelligence and Neuroscience, vol. 2016, pp. 1-16, 2016. doi: https://doi.org/10.1155/2016/6708183

[28] K. G. Liakos, P. Busato, D. Moshou, S. Pearson, and D. Bochtis, "Machine learning in agriculture: A review," Sensors, vol. 18, no. 8, pp. 1-29, 2018. doi: https://doi.org/10.3390/s18082674

[29] F. Recknagel, "Applications of machine learning to ecological modelling," Ecological Modelling, vol. 146, no. 1-3, pp. 303-310, 2001. doi: https://doi.org/10.1016/S0304-3800(01)00316-7

[30] V. Tatarinov, A. Manevich, and I. Losev, "A system approach to geodynamic zoning based on artificial neural networks," Gornye nauki i tekhnologii= Mining Science and Technology (Russia), vol. 3, no. 3, pp. 14-25, 2018. doi: https://doi.org/ 10.17073/2500-0632-2018-3-14-25 
[31] C. Clancy, J. Hecker, E. Stuntebeck, and T. O'Shea, "Applications of machine learning to cognitive radio networks," IEEE Wireless Communications, vol. 14, no. 4, pp. 47-52, 2007. doi: https://doi.org/10.1109/MWC.2007.4300983

[32] N. M. Ball and R. J. Brunner, "Data mining and machine learning in astronomy," International Journal of Modern Physics D, vol. 19, no. 07, pp. 1049-1106, 2010. doi: https://doi.org/10.1142/S0218271810017160

[33] T. U. Daim, G. Rueda, H. Martin, and P. Gerdsri, "Forecasting emerging technologies: Use of bibliometrics and patent analysis," Technological Forecasting and Social Change, vol. 73, no. 8, pp. 981-1012, 2006. doi: https://doi.org/10. 1016/j.techfore.2006.04.004 